\title{
Reflets
}

Revue ontaroise d'intervention sociale et communautaire

\section{Les services intégrés pour les enfants du nord : une pratique socio-communautaire à notre image}

\section{Marie A. Turcotte}

Volume 1, numéro 1, printemps 1995

Des pratiques à notre image : défis et ressources

URI : https://id.erudit.org/iderudit/026059ar

DOI : https://doi.org/10.7202/026059ar

Aller au sommaire du numéro

Éditeur(s)

Reflets : Revue ontaroise d'intervention sociale et communautaire

ISSN

1203-4576 (imprimé)

1712-8498 (numérique)

Découvrir la revue

Citer cet article

Turcotte, M. A. (1995). Les services intégrés pour les enfants du nord : une pratique socio-communautaire à notre image. Reflets, 1(1), 166-174.

https://doi.org/10.7202/026059ar

Tous droits réservés (C) Reflets : Revue ontaroise d'intervention sociale et communautaire, 1995
Ce document est protégé par la loi sur le droit d'auteur. L'utilisation des services d'Érudit (y compris la reproduction) est assujettie à sa politique d'utilisation que vous pouvez consulter en ligne.

https://apropos.erudit.org/fr/usagers/politique-dutilisation/ 


\title{
Les services intégrés pour les enfants du nord: une pratique socio-communautaire à notre image
}

\author{
Marie A. Turcotte, M.s.s. ${ }^{1}$ \\ Travailleuse satellite, Services intégrés pour les enfants du Nord à Hearst
}

Entre 1980 et 1985, les intervenants sociaux du nord de l'Ontario reconnaissaient qu'un nombre considérable d'enfants de leur milieu ne recevaient pas les services dont ils avaient besoin dans les domaines de l'éducation, de l'enfance en difficulté et de la santé physique et mentale. Ces lacunes résultaient d'un ensemble de facteurs. En raison du nombre de ministères engagés dans la prestation des services pour ces jeunes, ceux et celles éprouvant des problèmes multiples pouvaient littéralement être perdus dans un dédale administratif. Certaines familles devaient parcourir de longues distances pour recevoir des services, puisque les organisations en place n'atteignaient pas ou très peu les collectivités rurales isolées. Souvent, ces services n'étaient pas appropriés, autant aux niveaux linguistique que culturel. De plus, en raison des grandes étendues qui séparent les principaux centres urbains du nord de l'Ontario, il était extrêmement difficile de recruter les professionnels souhaités et, une fois sur place, de les y retenir. 


\section{Historique du programme}

En juillet 1985, le Conseil des ministres du gouvernement de l'Ontario préconise une approche interministérielle afin d'améliorer le processus d'évaluation et la prestation de services aux enfants ayant des besoins particuliers dans le nord de la province. Par la suite, des représentants des ministères provinciaux des Services sociaux et communautaires, de l'Éducation et de la Santé forment des comités continus de gestion interministérielle dans chacun des trois secteurs principaux du nord (nord-ouest, nord-centre et nord-est). Ces trois comités de secteur ont comme premier mandat de revoir les cas jugés exceptionnels et de coordonner les évaluations spécialisées pour enfants ayant des besoins particuliers. De ce processus, deux éléments se dégagent clairement: la nécessité d'accroître le nombre de professionnels dans le domaine des services à l'enfance et celle d'assurer une meilleure coordination des services. Afin de répondre à ces besoins, le Conseil des ministres et le Conseil de direction donnent leur accord à une stratégie à long terme connue sous le nom de "Programme des initiatives du Nord en matière de services offerts aux enfants ayant des difficultés multiples».

L'annonce officialisant ce programme est faite le 28 avril 1988 par l'honorable John Sweeney, alors ministre des Services sociaux et communautaires. Un membre du personnel de ce ministère est retenu comme coordonnateur régional du programme en mai 1988 et trois coordonnateurs de secteur sont embauchés vers la fin de 1988 et au début de 1989. Le nouveau service, établi dans les six districts du nord de l'Ontario, prend le nom de Programme des Services intégrés pour les enfants du Nord (SIEN) en septembre 1989. Le programme sIEN adopte des procédures communes d'évaluation et de surveillance dès le début, selon un modèle orienté sur son utilisation.

Grâce à SIEN, les enfants des régions rurales et éloignées du nord de l'Ontario présentant des problèmes physiques, psychosociaux et scolaires jouissent maintenant d'un meilleur accès 
Reflets

aux services coordonnés de soutien professionnel qui sont désormais offerts aussi près que possible du lieu de leur domicile.

\section{Les objectifs et les modalités du programme}

Le programme SIEN emploie une méthode novatrice de prestation de services. En effet, celle-ci intègre les responsabilités traditionnelles relevant de plusieurs organismes des domaines de la santé et des services sociaux, en plus de réunir neuf disciplines professionnelles et quatre ministères provinciaux, soit ceux des Services sociaux et communautaires, de l'Éducation, de la Santé et du Développement du Nord et des Mines.

Le SIEN a pour mission de mettre en place un réseau intégré de services de qualité dans les domaines de la santé globale et de l'éducation de l'enfance en difficulté pour les enfants et leur famille des collectivités rurales isolées, situées à l'extérieur des six plus grands centres urbains du nord de l'Ontario (Timmins, North Bay, Sudbury, Sault Ste. Marie, Thunder Bay et Kenora). Les services tentent de respecter le plus possible la langue et la culture des personnes à qui ils s'adressent. Ils sont aussi organisés de manière à tirer le meilleur parti possible de la coopération et de la coordination des efforts interorganismes, interdisciplinaires et interministériels.

La gestion courante des services est assurée par un comité régional composé de directeurs d'agences de la région et de représentants des ministères engagés. Dans les communautés locales, la gestion est confiée aux structures administratives déjà en place, généralement les Centres de santé mentale pour enfants. Dans le centre urbain de chacune des six régions, un groupe de professionnels comprenant généralement un ou une psychiatre, orthophoniste, ergothérapeute, psychologue, psychométricienne et deux enseignants-diagnosticiens forme une équipe interdisciplinaire offrant des services d'évaluation, de consultation, de planification de traitement ou d'intervention directe et de for- 
mation. Les membres de l'équipe se rendent dans les différentes localités à diverses intervalles, selon la discipline et le mandat. L'équipe maintient un contact régulier avec les collectivités rurales isolées par l'intermédiaire d'un réseau de travailleurs et travailleuses satellites. Ces personnes, qui ont une formation en service social ou en psychologie, reçoivent les demandes de service, font la cueillette des données préliminaires et coordonnent les services directs ou indirects offerts aux enfants. Elles remplissent leurs fonctions sous la double direction de leur organisme hôte et du chef d'équipe de programme siEN de leur district. Elles constituent le seul moyen d'accès aux services offerts par SIEN, prévenant ainsi toute forme de dédoublement de service.

Lorsqu'un enfant est référé à l'équipe interdisciplinaire, cette dernière propose des modalités d'évaluation. Suite à ces évaluations, les professionnels des différentes disciplines établissent un plan d'intervention commun qui fera l'objet de révision à intervalles réguliers (3 mois, 6 mois, etc.).

La philosophie du programme s'inspirant d'un modèle de responsabilisation des parents (client responsive), ces derniers participent à l'énonciation des besoins de leur enfant, au choix de l'intervenant et à la détermination de la période de temps nécessaire pour l'évaluation, l'intervention et le suivi. Si, dans certains cas, les parents préferent déléguer cette tâche à l'équipe du SIEN, ils doivent déterminer de quelle façon ils veulent être informés ou prendre part aux décisions relatives à leur enfant. Les rapports d'évaluation ne sont fournis à d'autres agences (médecins, écoles, etc.) qu'avec leur consentement explicite. Ils doivent consentir au plan d'intervention et souvent même participer au suivi (p. ex. programmation en langage ou en motricité).

\section{Les succès de ce programme}

Tout au long de l'élaboration et de la mise en oeuvre des services, le partenariat entre les quatre ministères au plan financier, 
Reflets

administratif ou au niveau de la conception des services s'est avéré possible et viable, favorisant grandement la coordination des ressources. Les frontières administratives qui existaient entre ministères et organismes sont en train de disparaitre, pour laisser la place à un modèle holistique visant à assurer la continuité des soins. Cette intégration s'est aussi révélée moins coûteuse que la forme de services cloisonnés préalablement fournis par des établissements distincts, qui pouvait contribuer à certains dédoublements.

Le point d'accès unique pour les parents et les agences facilite le processus de référence. Une fois l'accueil complété, la travailleuse satellite s'informe auprès des agences impliquées dans le passé et peut demander des consultations extérieures, par exemple en audiologie ou en neurologie. Elle peut aussi organiser des études de dossiers «interagences». Les familles n'ont plus à parcourir de grandes distances pour recevoir les services puisque l'équipe de ressources spécialisées se rend dans leur région.

La gestion de cas est vue comme un élément important de l'intervention. Lynne Tessier, travailleuse satellite de l'équipe sIEN de Sudbury, dirige présentement une étude ayant pour but de valider l'importance et les habiletés nécessaires au rôle de coordination des ressources d'évaluation et d'intervention qu'assument les travailleuses satellites.

Le fait que les services de suivi puissent être offerts par des ressources autres que l'équipe de ressources professionnelles, tel les parents, bénévoles, aides-enseignantes, travailleurs de soutien, constitue aussi un des avantages de SIEN. Ceci permet aux intervenants de consacrer une plus grande proportion de leur temps aux services indirects comme la consultation, les conférences interagences, la supervision d'étudiants ou de para-professionnels, etc.

Certains projets pilotes ont aussi été mis sur pied. Dans la région de Cochrane nord et de Cochrane sud, des services d'intervention directe en ergothérapie, physiothérapie et orthophonie sont offerts à chaque semaine par une assistante résidant dans le milieu et cela, sous la supervision des spécialistes de santé de l'équipe de ressources professionnelles. 


\section{Défis rencontrés par le programme}

Plusieurs défis restent à être relevés puisque le programme SIEN est toujours en développement. Le manuel de politiques et procédures est constamment en révision. Certains points restent à négocier au niveau des ministères, par exemple, le fait que les intervenants du programme ne puissent se rendre sur les réserves bien que leurs écoles aient manifesté le désir de pouvoir bénéficier des services de SIEN.

Un grand défi concerne la demande toujours élevée de multiples évaluations spécialisées pour un même enfant (p. ex., éducationnelle, psychométrique, orthophonique, etc.). Certaines disciplines ont une liste d'attente très longue. Il faudra arriver à un meilleur équilibre entre la demande d'évaluations spécialisées et la capacité de fournir l'intervention et le suivi nécessaires. L'équipe tente de faire un dépistage plus serré au moment de l'accueil afin d'éviter les évaluations qui ne sont pas vraiment nécessaires. Certains critères retenus dans cette prise de décision sont l'âge de l'enfant et le manque de ressources ou d'intérêt pour faire suite aux recommandations. Il faut aussi accroitre la capacité d'intervention traditionnelle et non-traditionnelle de l'équipe.

Le problème lié au roulement du personnel constitue un autre défi de taille. Si un poste demeure vacant pendant plusieurs mois, toute l'intégration interdisciplinaire est affectée. Les distances à parcourir et le temps nécessaire pour les déplacements sont un facteur de stress pour les membres de l'équipe. Cependant, ils voyagent en groupe afin de palier à ce problème et peuvent ainsi en profiter pour discuter de questions professionnelles et, du même coup, favoriser une cohésion accrue.

Le maintien des services, malgré les coupures budgétaires draconiennes imposées par certains ministères, constitue un autre enjeu d'importance. Avec la réduction importante du personnel d'éducation spécialisée dans les écoles, la coordination des services (études de cas, suivi par les aides-enseignantes, etc.), s'avère de plus 
Reflets

en plus difficile. Lorsque l'enfant présente des problèmes multiples, incluant des difficultés émotives ou familiales, nous devons le diriger vers les Centres de santé mentale pour enfants. Il y a alors une certaine duplication de services relativement aux questionnaires d'accueil (histoire développementale, médicale, scolaire) et aux formulaires de consentement des agences impliquées.

Vu le nombre élevé de clients dans chaque région, il reste peu de temps pour des activités de prévention (p. ex., ateliers pour les parents) et pour l'implication communautaire (p. ex., comité pour l'Année de la Famille, forum communautaire pour l'intégration des services, etc.). On souhaiterait que ceci devienne possible à mesure que les questions administratives exigeront de moins en moins de temps du personnel en place dans les diverses localités.

Enfin, un défi de taille pour nos services est le nombre élevé de clients éprouvant des problèmes multiples et cela sur une longue période. Par exemple, les enfants avec handicaps physiques ou avec troubles d'apprentissage ou de langage sérieux exigent de notre programme des services à long terme. Nous essayons de répartir le mieux possible cette clientèle entre les différentes catégories selon les besoins les plus pressants (court terme actif, suivi périodique ou suivi à long terme).

\section{Les perspectives d'avenir}

Plusieurs éléments nous permettent de croire à un meilleur fonctionnement des Services intégrés pour les enfants du Nord au cours des années à venir. Une restructuration des niveaux de gestion interministérielle est actuellement considérée. Il existe une possibilité d'éliminer un palier intermédiaire. Ce changement dans la structure administrative faciliterait d'autant plus la communication et la prise de décisions. La consultation de la population locale pourrait se faire par le biais d'un comité local consultatif dans chaque communauté dans un avenir prochain. SIEN deviendrait un partenaire à part égale au sein de ce comité. 
Ceci permettrait de mieux définir les besoins spécifiques de chaque localité et favoriserait l'intégration des services.

Suite aux rapports d'évaluation qui ont été effectués l'an dernier, nous espérons voir une redistribution des ressources humaines et économiques en tenant compte des analyses statistiques et des besoins de chaque communauté. Les facteurs dont on devrait tenir compte incluent, entre autres, le nombre de clients, la distribution géographique, le pourcentage d'enfants à problèmes multiples relevant de la juridiction de plusieurs ministères dans chaque région de même que la chronicité de leur état.

Il serait souhaitable qu'on apporte des éclaircissements relativement à notre rôle vis-à-vis les réserves indiennes. Plus précisément, il nous paraît désirable qu'on donne à sIEN le mandat de s'impliquer directement dans les réserves auprès des enfants autochtones. Idéalement, une équipe sIEN devrait être établie spécifiquement pour desservir les besoins des réserves du grand Nord-ontarien. On pourrait former des travailleurs autochtones à cette fin.

\section{En conclusion}

Le mouvement actuel d'intégration des services pour la famille et l'enfance est très prometteur. Le modèle du document Les enfants d'abord est en quelque sorte le concept élargi du programme SIEN, en prévoyant y inclure d'autres ministères et d'autres services, tels que les Services à la Jeunesse, une maison pour femmes abusées, les services familiaux, etc. Un projet pilote est en cours à Hearst en vue de former un comité qui s'occupera du suivi du forum communautaire portant sur l'intégration des services aux enfants et à leurs familles.

L'intérêt porté au programme sIEN s'étend à toute la province surtout dans les centres urbains du sud et de l'est de l'Ontario et au niveau international. L'été dernier, nous avons eu la chance de recevoir une équipe internationale venue étudier notre fonctionnement. 
Reflets

Cette délégation de l'OCDE (Organisation de coopération et de développement économique) et du Centre pour la recherche et l'innovation dans l'enseignement regroupait des représentants de plusieurs pays. Les conclusions tirées de leurs observations apparaitront dans leur rapport final qui doit être publié d'ici la fin de l'année.

\section{Note}

1. Employée au Services à la jeunesse Jeanne Sauvé à Hearst. Article revu par Claudette Lamontagne, B.s.s.. directrice du Programme des services intégrés pour les enfants du Nord pour les 3 districts de Témiscamingue, Cochrane nord et Cochrane sud. Mme Lamontagne est employée au Services intégrés pour les enfants du Nord à Timmins.

\section{Bibliographie}

COMITÉ CONSULTATIF SUR LES SERVICES À L'ENFANCE (1990). Les enfants d'abord, Imprimeur de la Reine pour l'Ontario. 